\title{
XXVII. On obliquely-crossed cylindrical lenses
}

\section{Silvanus P. Thompson D.Sc. F.R.S.}

To cite this article: Silvanus P. Thompson D.Sc. F.R.S. (1900) XXVII. On obliquelycrossed cylindrical lenses, Philosophical Magazine Series 5, 49:298, 316-324, DOI: 10.1080/14786440009463849

To link to this article: http://dx.doi.org/10.1080/14786440009463849

$$
\text { 曲 Published online: } 21 \text { Apr } 2009 .
$$

Submit your article to this journal $\sqsubset$

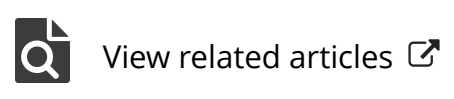

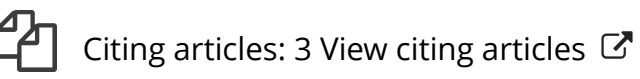


menon, together with the curves for the E.M.F. of various couples during henting and cooling - thermo-electric hysteresis curves as they may be called*. It is very probable that the peculiar thermo-electric deportment of iron, and some of the alloys of iron described in this paper, is intimately associated with the phenomenon of recalescence, or rather of the series of recalescent points which exist in iron and steel.

XXVII. On Obliquely crossed Cylindrical Lenses. By Silvanus P. Thompson, ji.Se., F.R.S. $\dagger$

CYLINDRICAL lenses have not claimed much attention $U$ from writers on geometrical optics. Certain of their properties, which make them invaluable to the ophthalmic surgeon for the correction of astigmatism, are, however, considered in all modern treatises on ophthalmics. Airy applied them for this purpose, but Donders first treated them systematically, in 1862, in his work Astigmatismus und cylindrische Gläser. Reusch in 1868 published his Theorie der Cylinderlinsen. Javal has written much on the subject from the ophthalmological point of view, as has Mr. S. M. Burnett. Mr. C. F. Prentice has also written of them in two works, a 'Treatise on Ophthalmic Lenses' and 'Dioptric Formulæ for combined Cylindrical Lenses.' Stokest has proposed a cylindrical lens of variable power by combining at a variable angle two equal cylindrical lenses of opposite sign. The problem of the optical properties of crossed cylindrical lenses was touched upon by Reusch and by Donders. Krüss has written upon the aberration of "bicylindrical" lenses, meaning by that term such lenses as have both surfaces cylindrical and of equal curvature, but with their axes mutually at right-angles. Lastly, Prentice has given very elaborate rules for calculating the equivalent sphero-cylindrical lens for any combination of two lenses crossed at any angle. The importance of the solution of this problem arises from a point in modern ophthalmic practice:In cases of ordinary astigmatism in which the refractive

* As was pointed out by Professor G. F. FitzGerald, F.T.C.D., F.R.S., at the meeting of the Society when this paper was read, the thermoelectric hysteresis here referred to is, no doubt, the cause of the thermocurrent which is produced in an iron wire steadily moved through a flame, a phenomenon first noticed and investigated by Dr. F. I. Trouton, F.R.S. See Proc. Roy. Dublin Society, March 1886. I am also greatly indebted to Professor FitzGrerald for other suggestions he has made in reading the proof of this paper.

† Communicated by the Physical Society : read Dec. 8, 1899.

\$ Mathematical and Physical Papers, vol. ii. p. 172. 
power of the eve is different in different meridians around the optic axis. One process of examination of this defect consists in ascertaining the position of the meridian of greatest refractive power, and in then measuring that power; and, this having been done, measuring the power in a meridian at right angles to the former, that is to say in the meridian of minimum refractive power. The difference between these two powers gives in reality the amount of cylindricity to be corrected. Or the excess of each over the normal spherical refractive power of the eye might be separately corrected by the choice of two appropriate cylindrical lenses which are then superposed at right angles. Sometimes, however, ophthalmic surgeons, whether through insufficient appreciation of the geometrical and optical principles involved, or through some incidental cause, prescribe a lens with two cylindrical curvatures on the respective faces of the lens, not crossed at right-angles but at some oblique angle. As such lenses are difficult of manufacture, and as their optical effect can be precisely reproduced by a suitably calculated and more readily ground sphero-cylindrical lens, the optician desires to have simple rules for calculating the equivalent sphero-cylinder. Hence the present attempt to arrive at easier rules for obliquelycrossed cylindrical lenses. To establish these rules it is possible to proceed by a simpler method than that of Reusch, whose investigation is exclusively based upon the properties of parabolic lenses.

2 . In the case of thin cylindrical lenses it is customary to call a line drawn through the lens, in a direction parallel to the axis of the generating cylinder of which its curved surface forms a part, "the axis" of that cylindrical lens. Let there be two thin cylindrical lenses placed in contact behind one another,

Fig. 1.

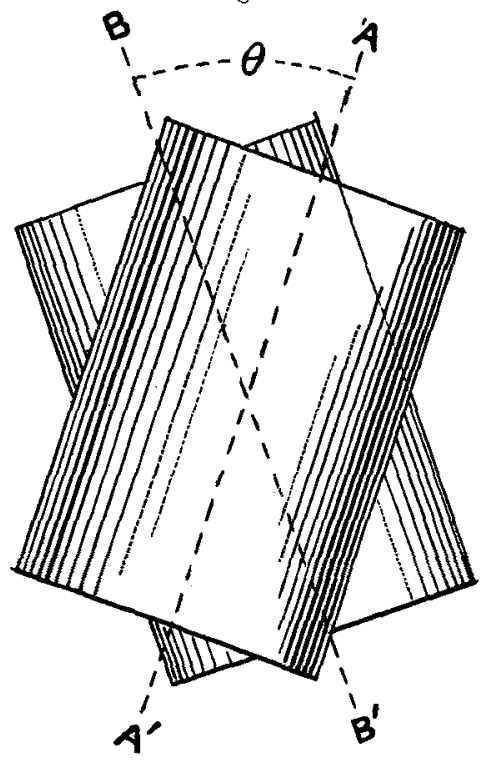
so that their axes $\mathrm{A} \mathrm{A}^{\prime}, \mathrm{B} \mathrm{B}^{\prime}$ make an angle $\theta$ with on $\theta$ another. The optical axis of the system passes through their intersection and is normal to the plane containing them. 
It is required to find the combination consisting of one thin cylindrical lens and one spherical lens which will be the optical equivalent of the system; or, the sphero-cylindrical lens that is also equivalent. The problem is completely solved when we shall have ascertained the two components, cylindrical and spherical, of the equivalent lens and the angular position of the cylindrical component.

In any lens having at one surface a radins of curvature $r$, the curvature which that surface will impress upon a plane wave is $(\mu-1) / r$; where $\mu$ is the refractive index of the material. If the lens is cylindrical, having a curvature in one meridian only, the impressed curvature will also be cylindrical; if it be spherical, the impressed curvature of the wave-front will be correspondingly spherical. First approximations only are here considered.

Let (fig. 2) $\mathrm{A} \mathrm{A}^{\prime}$ be the axis of a cylindrical lens, and

Fig. 2.

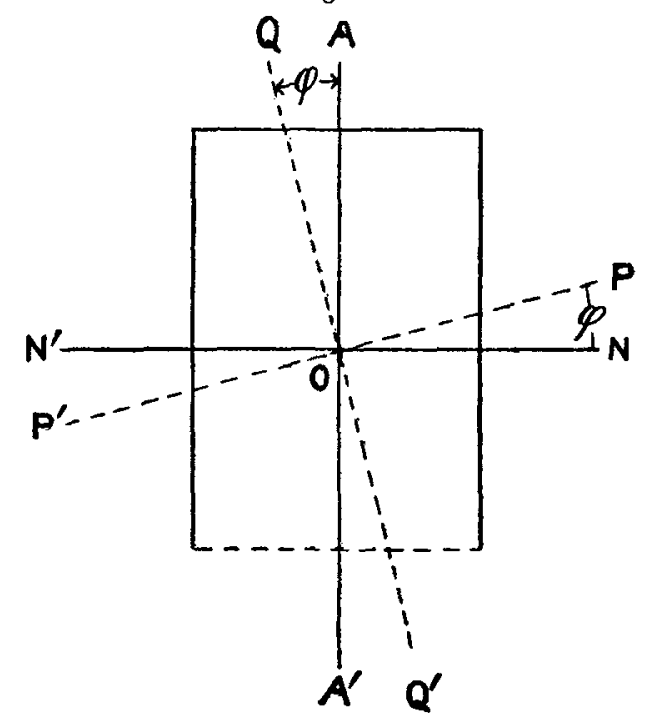

$\mathrm{N} \mathrm{N}^{\prime}$ a line normal to that axis. A plane normal to the axis intersecting the lens in $\mathrm{NN}^{\prime}$ will have as its trace through the curved surface of the lens a line of the same curvature as the lens, viz. $\frac{1}{r}$. Let now an oblique intersecting plane be drawn through the optic axis of the system $(i . e$. the line through $\mathrm{O}$ normal to the plane of the diagram); its intersection $\mathrm{PP}^{\prime}$ making an angle $\mathrm{N} O \mathrm{P} \equiv \phi$ with the line $\mathrm{N}^{\prime}$. The 
curvature at $O$ of the trace of this plane, where it cuts the curved surface along $\mathrm{P} \mathrm{P}^{\prime}$, will be $\frac{1}{r} \cos ^{2} \phi$. This follows from the circumstance that $\mathrm{P} \mathrm{P}^{\prime}$ is part of an ellipse whose major and minor axes are respectively $r / \cos \phi$ and $r$. We may further consider the intersection $\mathrm{Q} \mathrm{Q}^{\prime}$ of another oblique plane at right-angles to $\mathrm{P}^{\prime}$. The curvature at 0 along the line $\mathrm{Q}^{\prime}$ will be $\frac{1}{r} \sin ^{2} \phi$. The sum of these two curvatures will obviously be equal to the original maximum curvature along $\mathrm{N} \mathrm{N}^{\prime}$, since the minimum curvature along $\mathrm{A}^{\prime}$ is zero. Hence we may regard the curvature along $\mathrm{N} \mathrm{N}^{\prime}$ as contributing two components of cylindricity of the respective values named in the two directions $P P^{\prime}$ and $Q Q^{\prime}$. If light were admitted through narrow parallel slits set respectively along $P \mathbf{P}^{\prime}$ and $\mathrm{Q}^{\mathrm{Q}} \mathrm{Q}^{\prime}$, the convergivity of the two beams respectively impressed by the lens would be $(\mu-1) \cos ^{2} \phi / r$ and $(\mu-1)$ $\sin ^{2} \phi / r$. If $r$ is expressed in metres, these two convergivities will be expressed in dioptries according to the practice now internationally adopted by ophthalmists. It is obvious that the angle $\phi$ may be measured either between $\mathrm{N} \mathrm{N}^{\prime}$ and $\mathrm{P} \mathrm{P}^{\prime}$, or between $A A^{\prime}$ and $Q Q^{\prime}$.

3. Returning to the problem enunciated with reference to fig. 1, we may now find a solution by resolving each of the two cylindrical lenses into components and then recombining these components in the manner presently to be considered.

Let the line $\mathrm{O} a$ (fig. 3 ) represent the direction of the axis of one of the given cylindrical lenses, having a power of $\mathrm{A}$ dioptries, and the line $O b$ the direction of the axis of the other given cylindrical lens of power $\mathrm{B}$ dioptries. The angle $\theta$ between $\mathrm{O} a$ and $\mathrm{O} b$ is also given. It is required to find the respective number of dioptries $\mathrm{C}$ and $\mathrm{D}$ of the cylindrical and spherical lenses which shall together constitute a combination whose optical effect is the equivalent of that of $\mathrm{A}$ and $\mathrm{B}$. It is also required to find the angular position of

Fig. 3.

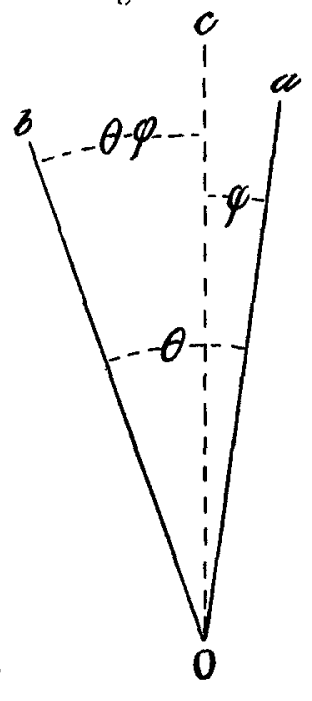
the axis of the equivalent cylindrical lens.

It is clear that we might take any line $\mathrm{O} c$ through $\mathrm{O}$ making an angle $\phi$ with $O a$, and take the cylindrical components, along that direction, of the two given cylindrical 
lenses $A$ and $B$. The sum of these components would be $\mathrm{A} \cos ^{2} \phi+\mathrm{B} \cos ^{2}(\theta-\phi)$. Similarly there might be taken, at right-angles to $\mathrm{O} c$, the two other cylindrical components, whose sum would be $A \sin ^{2} \phi+B \sin ^{2}(\theta-\phi)$. Now there will be in every case one particular value of $\phi$ which will make the former sum a maximum and the latter a minimum. If we can find this value of $\phi$, the problem is solved.

Differentiating with respect to $\phi$ the expression above obtained for the sum of the two components along $\mathrm{O} c$, and equating to zero, we find that when this sum is a maximum the angle $\phi$ will be such as to give the relation

$$
\widetilde{\mathrm{B}}=\frac{\sin 2(\theta-\phi)}{\sin 2 \phi}, \ldots . \quad . \quad \text {. }
$$

to which may be given the alternative form

$$
\cot 2 \phi=\frac{\overline{\mathrm{B}}+\cos 2 \theta}{\sin 2 \theta} . \quad . \quad . .
$$

From this latter $\phi$ may be reckoned by the aid of trigonometrical tables, A, B, and $\theta$ being all given. Angle $\phi$ being thus found, it can be used to calculate the maximum and minimum values, namely the two sums previously expressed. The cylindrical lens representing the maximum sum being set at angle $\phi$ with the original direction of $A$, and the eylindrical lens representing the minimum sum being set at $90^{\circ}-\phi$, they will, thus crossed at right-angles to one another, together act as the optical equivalent of the two obliquelycrossed cylinders.

These two rectangularly-crossed cylindrical lenses may again be resolved into the combination of (1) a cylindrical lens, whose axis is along the axis just found for the maximum lens, and of power $\mathrm{C}$ dioptries equal to the difference between the maximum and minimum cylindrical powers, and (2) a spherical lens whose power $D$ is that of the minimum. Hence we shall have and

$$
\begin{aligned}
& \mathrm{C}=\mathrm{A} \cos ^{2} \phi+\mathrm{B} \cos ^{2}(\theta-\phi)-\mathrm{A} \sin ^{2} \phi-\mathrm{B} \sin ^{2}(\theta-\phi) \\
& \mathrm{C}=\mathrm{A} \cos 2 \phi+\mathrm{B} \cos 2(\theta-\phi), \quad . \quad . \quad . \quad . \quad . \quad . \quad(3)
\end{aligned}
$$

$$
\mathrm{D}=\mathrm{A} \sin ^{2} \phi+\mathrm{B} \sin ^{2}(\theta-\phi) \text {. }
$$

4. The solution thus found is capable of further simplification.

Dividing equation (3) by $\mathrm{A}$, we have :

$$
\frac{\mathrm{C}}{\mathrm{A}}=\cos 2 \phi+\frac{\mathrm{B}}{\overline{\mathrm{A}}} \cos 2(\theta-\phi) \text {. }
$$


From equation (1) we have

$$
\frac{B}{A}=\frac{\sin 2 \phi}{\sin 2(\theta-\phi)} .
$$

Substituting this in the preceding gives us :

$$
\begin{aligned}
& \frac{\mathrm{C}}{\mathrm{A}}=\cos 2 \phi+\frac{\sin 2 \phi \cdot \cos 2(\theta-\phi)}{\sin 2(\theta-\phi)}, \\
& \frac{\mathrm{C}}{\mathrm{A}}=\frac{\sin 2 \theta}{\sin 2(\theta-\phi)} .
\end{aligned}
$$

From this immediately follows the relation :

$$
\frac{A}{\sin 2(\theta-\phi)}=\frac{B}{\sin 2 \phi}=\frac{C}{\sin 2 \theta^{\circ}} \quad . \quad .
$$

This at once suggests that the three magnitudes $A, B$, and $\mathrm{C}$ can be represented by the three sides of a triangle whose respectively-subtended angles are $2(\theta-\phi), 2 \phi$, and $2 \theta$. Or $2(\theta-\phi), 2 \phi$, and $\pi-2 \theta$. In short, the two given cylindrical components $A$ and $B$ may be compounded to find their cylindrical resultant $\mathrm{C}$ by means of a parallelogram in which, however, the angle between $\mathrm{A}$ and $\mathrm{B}$ is drawn as double the actual angle between the axes of the two given components.

Hence we obtain the graphic construction of fig. 4. Draw the lines O A, O B to represent, in magnitude only, the powers of the two given cylindrical lenses, and make the angle $\mathrm{AOB}$ between them double the given angle $\theta$. For positive (i. e. convergent) cylindrical lenses these directions may be reckoned outwards from the origin, as shown by the arrow-heads. For negative (i. e. divergent) cylindrical lenses the directions must be reckoned inwards. Compound-ing $\mathrm{OA}$ and $\mathrm{OB}$ in the ordinary way, we obtain the resultant $\cup \dot{C}$ which represents in magnitude (and in sign) the resultant cylindrical part of the desired equivalent combination; but the angle $\mathrm{A} \mathrm{O} \mathrm{C}$ will be double of the angle that the axis of Fig. 4.

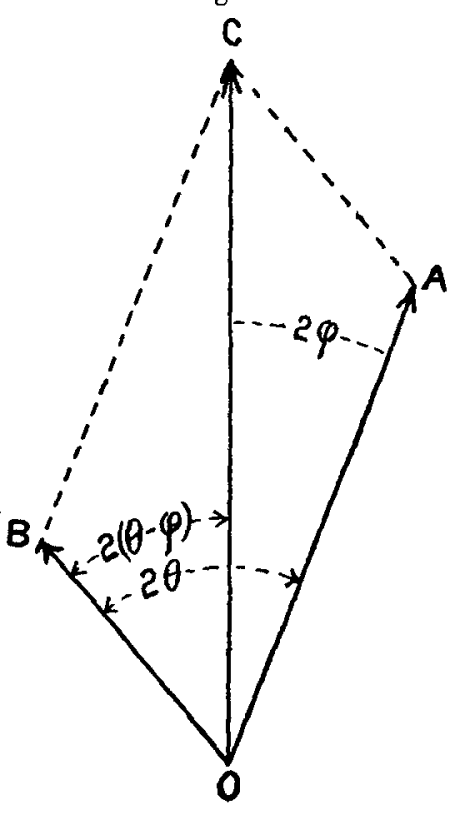
the resultant cylindrical lens will make with the axis of $A$. 
It will then be evident that the value of $\mathrm{C}$ is at once calculable directly from $A, B$, and $\theta$ by the equation

$$
\mathrm{C}^{2}=\mathrm{A}^{2}+\mathrm{B}^{2}+2 \mathrm{AB} \cos 2 \theta \text {. }
$$

Having obtained $\mathrm{C}$, the angle $\phi$ can be most easily calculated by the relation

$$
\sin 2 \phi=\frac{\mathrm{B}}{\mathrm{C}} \sin 2 \theta . \quad \text {. . . . . }
$$

It only remains to find the corresponding expression for the power $D$ of the spherical part of the equivalent combination.

Dividing equation (4) by A we have

$$
\begin{aligned}
\frac{\mathrm{D}}{\mathrm{A}} & =\sin ^{2} \phi+\frac{\mathrm{B}}{\mathrm{A}} \sin ^{2}(\theta-\phi) . \\
& =\frac{1}{2}\left\{\frac{\sin 2(\theta-\phi)+\sin 2 \phi-\sin 2 \theta}{\sin 2(\theta-\phi)}\right\} \\
& =\frac{1}{2}\left\{1+\frac{\mathrm{B}}{\mathrm{A}}-\frac{\mathrm{C}}{\mathrm{A}}\right\} \\
& =\frac{1}{2} \frac{\mathrm{A}+\mathrm{B}-\mathrm{C}}{\mathrm{A}},
\end{aligned}
$$

whence

$$
\mathrm{D}=\frac{\mathrm{A}+\mathrm{B}-\mathrm{C}}{2} \text {. . . . . . . . . . . }
$$

This last result might also bave been obtained by remembering that the maximum power being $\mathrm{C}+\mathrm{D}$ and the minimum power being $\mathrm{D}$ (at right angles), the sum of these, namely $\mathrm{C}+2 \mathrm{D}$, will be equal to $\mathrm{A}+\mathrm{B}$, whatever the angle between the lattor.

An example of the use of the three working formulæ (6), (7), and (8) will suffice.

To find the equivalent sphero-cylindrical combination for the following obliquely-crossed cylindrical lenses :-

Here

$$
+3.5 \text { cyl. ax. } 20^{\circ} \bigodot+2.5 \text { cyl. ax. } 35^{\circ} \text {. }
$$

$$
\begin{aligned}
\mathrm{A} & =3.5 \text { dioptries, } \\
\mathrm{B} & =2.5 \text { dioptries, } \\
\theta & =15^{\circ}, \\
\cos 2 \theta & =0.866, \\
\sin 2 \theta & =0.5,
\end{aligned}
$$


Obliquely-crossed Cylindrical Lenses.

By equation (6)

$$
\begin{aligned}
\mathrm{C}^{2} & =12 \cdot 25+6 \cdot 25+(17 \cdot 5 \times 0 \cdot 866) \\
& =33 \cdot 65,
\end{aligned}
$$

whence $\quad \mathrm{C}=+5 \cdot 8$ dioptries, approximately.

By equation (8)

$$
\begin{aligned}
& \mathrm{D}=\frac{3 \cdot 5+2 \cdot 5-5 \cdot 8}{2} \\
& \mathrm{D}=+0 \cdot 1 \text { dioptries (spherical) }
\end{aligned}
$$

By equation (7)

$$
\begin{aligned}
\sin 2 \phi & =\frac{2 \cdot 5}{5 \cdot 8} \times 0.5 \\
& =0 \cdot 215, \\
\phi & =\frac{1}{2} \sin ^{-1}(0 \cdot 215) \\
& =6^{\circ} 13^{\prime}, \text { approximately, beyond the position } \\
& \quad \text { of } \mathrm{A} .
\end{aligned}
$$

Hence the equivalent combination would be

$$
+0 \cdot 1 \mathrm{sph} . \bigodot+5 \cdot 8 \mathrm{cyl} \text {. ax. } 26^{\circ} 13^{\prime}
$$

5. The circumstance that the cylindrical part of the resultant of two crossed cylindrical lenses varies from maximum to minimum when the angle between the axes of the two lenses is varied from $0^{\circ}$ to $90^{\circ}$, suggests a solution to the practical problem how to make a cylindrical lens of variable cylindricity. If two equal positive cylindrical lenses are used, the value of the cylindrical part of their resultant varies from their sum, when the angle $\theta=0^{\circ}$, to zero when the angle $\theta=90^{\circ}$. But while the cylindrical part thus diminishes, in proportion to the square of the cosine of the angle between them, the spherical part of the resultant increases in the proportion of the square of the sine of the same angle. One never obtains a simple cylindrical lens, except in the case of the coincidence of the axes. Similarly if two equal negative cylindrical lenses are used, the resultant consists of a negative cylindrical part and a negative spherical part, the one decreasing, the other increasing when the angle $\theta$ is increased from $0^{\circ}$ to $90^{\circ}$.

Ophthalmists are acquainted with a combination (known as Stokes's lens) consisting of two cylindrical lenses of equal but opposite powers (one a convex, the other an equal concave), arranged to be rotated to various angles of obliquity across one another. When their axes are in coincidence, or $\theta=0^{\circ}$, they neutralize one another completely. When crossed at right-angles their resultant cylindricity is a 
maximum but is mixed in sign. Suppose their respective powers were +2 dioptries and -2 dioptries; they will when crossed not act as either a +4 dioptrie cylinder, or as a -4 dioptrie cylinder, but can be made to act as such by adding at choice either a +2 dioptrie spherical lens or $a-2$ dioptrie spherical lens, to neutralize the refractive effects in one or other of the meridians. When crossed at intermediate angles the spherical lens required to neutralize, and so produce the effect of a simple cylinder, would be of some intermediate power. This is a slight inconyenience; but a more serious one exists in the circumstance that in rotating one about the other the axis of the resultant cylindrical effect takes a varying obliquity. This objection is not removed by rotating the two lenses in opposite directions simultaneously.

A more convenient combination to give varying degrees of cylindricity is the following:-Let two lenses be ground, each being a mixed equi-cylinder consisting of a concave and convex ground at right-angles to one another on the opposite faces of the glass. Two such mixed cylinders, if rotated with equal motion in opposite directions, will give a varying cylindricity of fixed direction in space. With the axes of + cylindricity coincident they give the maximum; but when each is rotated to $45^{\circ}$, one to the right, the other to the left, their resultant is zero. In intermediate positions the value of the resultant varies according to the square of the cosine of double the angle through which either has been moved (i.e. to the square of the cosine of the angle between their axes), but it remains fixed in direction. When each is rotated beyond $45^{\circ}$ they begin again to act as a cylindrical lens, but with the resultant axis of cylindricity negative in the fixed direction in which formerly it was positive. An instrument thus constructed may be graduated so as to be direct-reading.

XXVIII. On the Law of Reciprocity in Diffuse Reflexion. By Lord RAYLeigh, F.R.S.*

T the current number of the Philosophical Magazine 1 (vol. xlix. p. 199) Dr. Wright discusses the question of the amount of light diffusely reflected from a given area of a matt surface as dependent upon the angle of incidence (i) and the angle of emission (c). According to Lambert's law the function of $i$ and $\epsilon$ is

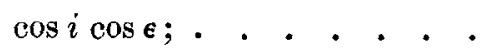

and this law, though in the present case without theoretical

* Communicated by the Author. 